\begin{abstract}
The role of microwaves is discussed in achieving the future wireless personal communications (FWPC) system: An overview of the FWPC system is presented and the challenges to meet its demands are examined. Special attention has been paid in defining the research challenges in the light of microwave perspectives. Among several research challenges, the key challenges are briefly described, such as propagation modeling, antenna diversity and millimeter waves. The description of their potentialities narrates the state of the art of future wireless personal communications. It outlines some of the prospective evolution of FWPC systems and expects to draw the research interest of the microwave community in the creation of a "Global Village."

\section{Overview of Wireless Personal Communications: Microwave Perspectives}

\author{
Ramjee Prasad, Delft University of Technology
}

had a delightful time writing this article because it reminded me of my long experience in the field of electromagnetic theory and microwave technology at the Birla Institute of Technology, Ranchi, India [1-3]. I started my research activities in the field of wireless personal communications in 1988 at Delft University of Technology, The Netherlands $[4,5]$. Due to my experience in microwave and wireless personal communications I am of the opinion that "electromagnetic theory" is the gateway to "electronics and communications," and "microwave technology" is the founding stone of "modern communications."

If we look back in history, we find that wireless communications begin with:

- The postulates of electromagnetic waves by James Clerk

Maxwell during the 1860s in England

- The demonstrations of the existence of these waves by Heinrich Rudolf Hertz in the 1880 s in Germany

- The invention of wireless telegraphy by Guglielmo Marconi, born in Bologna on April 25, 1874, during the 1890 s, first in Italy and then in the United Kingdom [6, 7$]$

During the 1890 s eminent scientists like Jagdish Chandra Bose in India, Oliver Lodge in England, and Augusto Righi at Bologna University were busy in the study of the fundamental nature of electromagnetic waves. In September 1895, over 100 years ago, Marconi could say that he had a wireless telegraph system with a potentially useful range, unaffected by natural obstacles [7]. It was a matter of great pride for me to be in Bologna in September 1995 during the 25th European Microwave Conference, which was one of the peak scientific events in celebrating the centenary of this

This article was originally presented at the 25th European Microwave Conference, as the keynote opening address, in Bologna, Italy, September 1995. landmark achievement of Marconi, "Wireless at 100 Years."

The notion of transmitting information without the use of wires must have seemed to our ancestors only 100 years ago to be magic. Marconi made it possible. In 1896 the first patent for wireless communications was granted to Guglielmo Marconi in the United Kingdom. Since then a number of developments in the field of wireless communications have taken place, as can be seen in Table 1. It is to be noted that this article deals with wireless communications in terms of radio technology only. Other wireless technology, such as infrared (IR), is not discussed here.

In 1980 the cellular era started. Several developments and new technologies will take place during the 1990s and 2000s. The second section presents the evolution of future wireless personal communications (FWPC). The role of microwave technology in the evolution of wireless personal communications

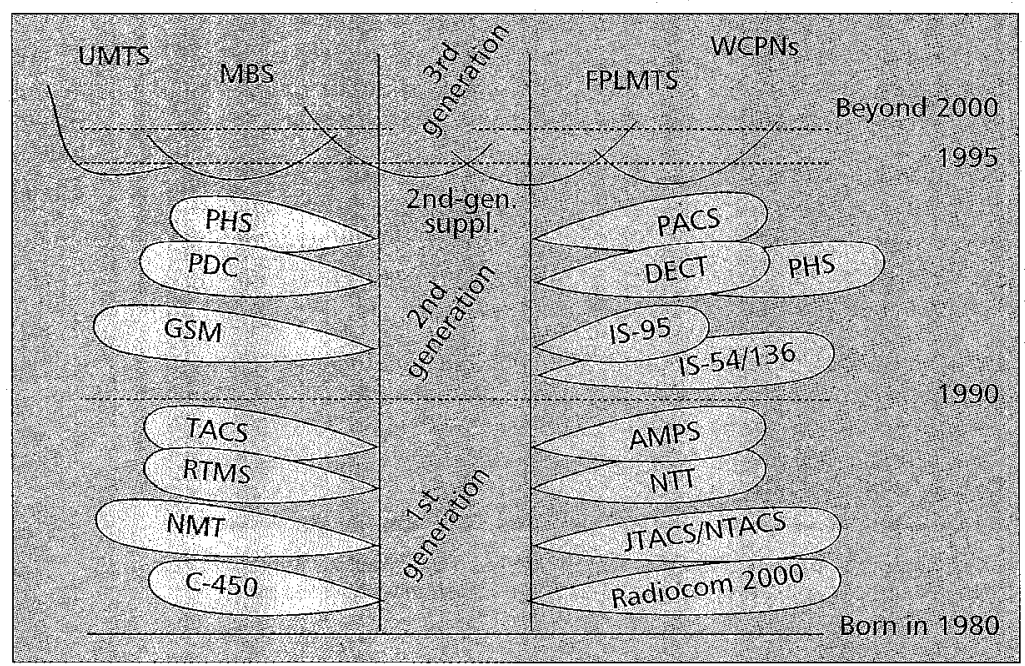

Figure 1. Family tree of wireless personal communications systems. 
is presented in the third section. Finally, conclusions are given in the last section.

\section{FWPC EVOLUTION}

$W$ th the current rapid growth of technology, it can now emphatically be said that the objective of today's communication engineers to achieve a future wireless personal communication (FWPC) system, which was yesterday's myth (before 1970), will be tomorrow's reality (beyond 2000). FWPC systems will convert the already shrinking world into a global village. An FWPC, defined as being the ultimate goal of today's communication engineers, will provide communication services from any person to any person in any place at any time without any delay in any form through any medium by using one pocket-sized unit at minimum cost with acceptable quality and security through the use of a personal telecommunication reference number [8].

A family tree for the FWPC system is shown in Fig. 1. The objectives of the research and development of FWPC systems are focused in three technological platforms: universal mobile telecommunication system (UMTSs), mobile broadband systems (MBSs), and wireless customer premises networks (WCPNs) [9]. UMTS is a multifunction, multiservice, multi-application digital system, evolving from currently operational second-generation systems and several other ongoing second-generation systems.

The first generation was introduced in 1980 in analog form to provide local mobile speech services and was then further extended to nationwide coverage. Various standard systems were developed worldwide: AMPS (Advanced Mobile Phone Service) in the United States, NTT (Nippon Telephone and Telegraph) systems in Japan, TACS (Total Access Communications System) in the United Kingdom, NMT (Nordic Mobile Telephones) in European countries, and so on.

Fast user growth was observed, penetrating up to 10 percent of the calls in North America, Western Europe, and Japan. The access technique used was frequency-division multiple access (FDMA). Capacity and quality were the major problems in the first-generation systems, as well as incompatible systems.

Advancements in digital technology gave birth to panEuropean digital cellular mobile (DCM) GSM (formerly Groupe Spécial Mobile, now Global System for Mobile Communications) systems in Europe, PDC (personal digital cellular) systems in Japan, and IS-54/136 and IS-95 in North America, which are the second-generation systems. Time-division multiple access (TDMA) is used as the access technique, except for IS-95, which is based on CDMA (code-division multiple access). The second-generation systems provide digi- tal speech and short message services. These services are expected to penetrate to more than 20 percent of the call population. GSM has become deeply rooted in Europe and several other countries worldwide. The development of new digital cordless technologies gave birth to the supplementary second-generation systems, namely, PHS (Personal Handyphone System, formerly PHP) in Japan, DECT (Digital Enhanced Cordless Telephone) in Europe, and PACS (Per sonal Access Communication Services) in North America. These may increase the call penetration depth up to 30 percent and introduce many new services. Although the second generation and its supplement will cover local, national, and international services, it will still have one major drawback in terms of a universal service facility.

The third-generation is expected to be deployed by the year 2000 via universal personal communications systems (UPCS), which will provide universal speech services and local multimedia services. It is expected that the third-generation system will penetrate up to 50 percent of the telecommunication services population. The third-generation PCSs are in the process of development worldwide by the ITU (International Telecommunications Union) within the framework of the 


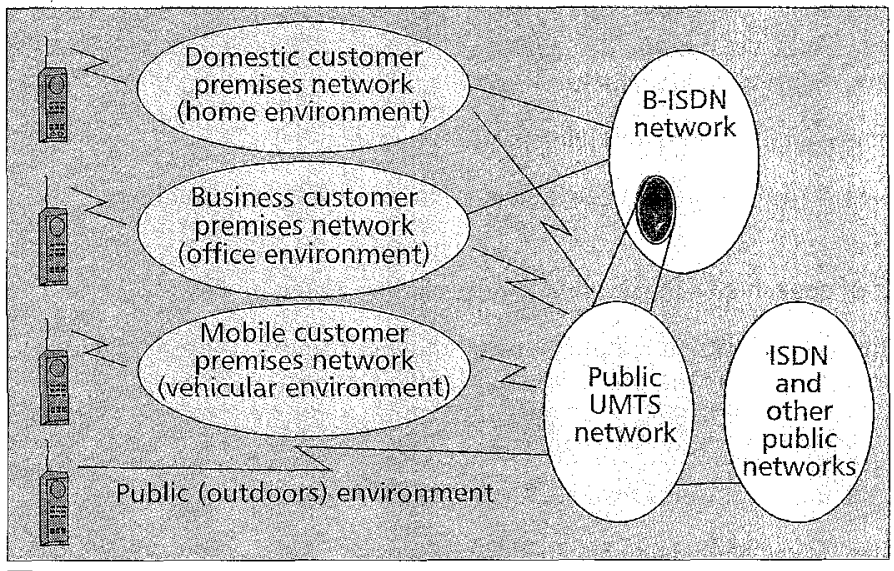

Figure 2. Possible UMTS service configuration.

FPLMTS (Future Public Land Mobile Telecommunications Systems)/IMT-2000 (International Mobile Telecommunications-2000) activities. In Europe this is supported by the UMTS program within the European Community. Both FPLMTS and UMTS programs are tightly related and expected to lead to consistent and compatible systems. Figure 2 shows the possible configuration for UMTS subnetworks and fixed networks.

UMTS is intended to provide a wide range of mobile services to users via a range of mobile terminals that enable the use of the pocket telephone in almost any location, indoor or outdoor, in home, office, or street. North America and Japan are also equally engaged in developing the third-generation systems in the UMTS direction. Looking at the research trends going on in Europe, Japan, and North America, it appears that the future access scheme will be either CDMA or one of the hybrid multiple access schemes based mainly on the combination of CDMA and TDMA/FDMA [8-10].

The only drawback will be the lack of rural (modern) communication coverage, particularly in developing countries. Hopefully, this will be solved at the beginning of the 21st century. Land-mobile satellite communications could solve this problem.

Figure 3 depicts a view of a global village integrating terrestrial pico-, micro-, and macrocellular systems and satellite networks. An integrated satellite-terrestrial cellular system is the concept of FWPC; that is, UPCS.

Cellular systems are classified into three categories, depending on the size of the cells, as follows:

- Macrocells of 2 to $20 \mathrm{~km}$ diameter with antenna radiating power in the range of 0.6 to $10 \mathrm{~W}$ from the top of tall buildings

- Microcells of 0.4 to $2 \mathrm{~km}$ diameter with antennas at streetlamp elevation and radiating power less than $20 \mathrm{~mW}$

- Picocells of much smaller size (20 to $400 \mathrm{~m}$ diameter) especially suited for indoor radio communications (e.g. offices, research laboratories, hospitals, modern factories, University campuses, etc.) with antennas placed on top of a bookshelf and radiating power on the order of a few $\mathrm{mW}$

Figure 4 shows the worldwide service evolution [9]. Universities, research laboratories, and industry are jointly busy exploring the following challenges to achieve the future wireless communications:

- Interfacing, internetworking and integration (ATM, fiber, air, fixed, pico-, micro-, macro,- and hypercells)

- Multiple access protocols (CDMA, TDMA, or hybrid?)

- System development (baseband, terminals, and antennas)

- Technology (low power, size, and cost)

- Higher frequency bands (62-63 GHz and 65--66 GHz millimeter waves)

- Mobile multimedia communications (MMC)
- Satellite (frequency allocation, channel characterization, radio access, etc.).

A significant advancement has been achieved in meeting these challenges which can be found in the published literature. It is worth mentioning that the European Community, Japan, and North America (the United States and Canada) have made firm decisions to respond to meet the challenges for FWPC systems through ambitious infrastructure investment programs and regulatory and legislative reforms. National communications and information networks are being introduced in these countries, attracting a continuous stream of innovative valued-added products and services. All governments have decided to spend billions of dollars for research and development in this field. Several challenging projects have started since September 1, 1995 in Europe within the Advanced Communications Technologies and Services (ACTS) program initiated by the European Commission.

\section{ROLE OF MICROWAVES}

Microwave technology is the "founding stone" in the evolution of FWPC. Since the invention of wireless, there has been a strong link between the progress of wireless communications and modern microwave techniques. It is worth noting that the most scientifically and technologically advanced applications of radio wave propagation currently take place in the microwave field. If we consider the challenges to meet the requirements for achieving the global village, microwave technology plays a major role in the following research areas.

Propagation Modeling - The knowledge of propagation modeling of a wireless channel is bread and butter for the wireless engineers. If the propagation modeling of the channel is not well developed, an efficient communications system can never be achieved; that is, we can never fulfill the dream of a global village.

In order to model a wireless channel, the following steps are taken:

- Develop the measurement setup.

- Conduct the narrow- and wideband measurements.

- Predict the model.

- Characterize the channel.

All these steps can be successfully completed with broad knowledge of electromagnetic theory and microwave technology. It is necessary to understand free space propagation, uniform

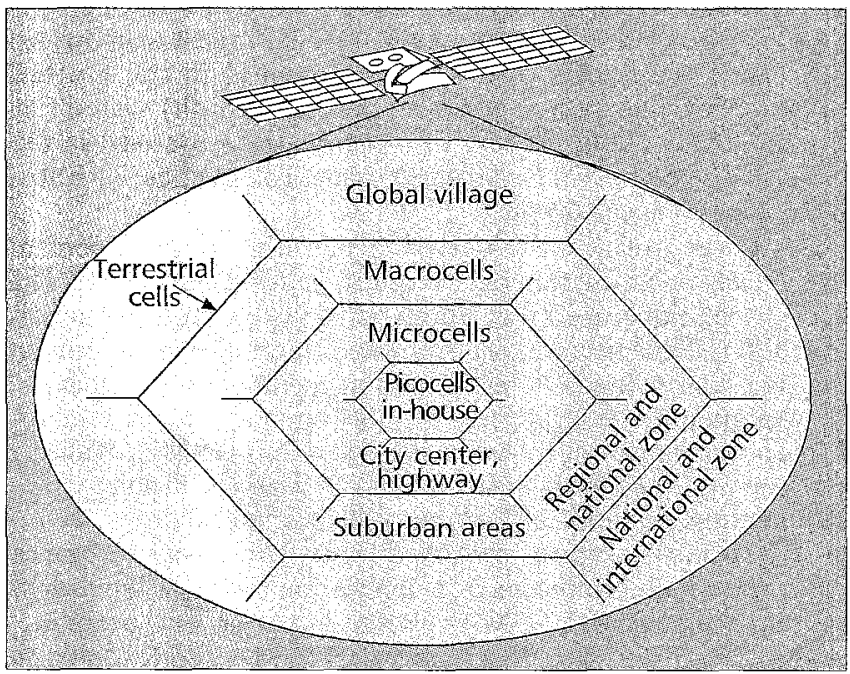

Figure 3. View of a future global village. 
plane wave reflection and transmission, propagation over flat earth, diffraction, scattering from rough surfaces, ray modeling, and antenna theory [11-13].

Antennas and Diversity - A critical knowledge of the antenna theory is necessary for the development of a wireless communication system. The antennas are the outermost components in the transmission chain. The behavior of an antenna depends on the interaction between the antenna and the wireless environment. The wireless environment is very hostile in nature, which is typified by a multiple scattering process and the absence of direct line-of-sight paths between the base and the mobile. The conventional free-space antenna pattern is thus greatly modified, and antenna designs have to be tailored to the statistical nature of the environment [12]. The primary task of the antenna is to convey the information from transmitter to receiver. However, it is also of equal importance to consider the antenna for suppression or repair of media impairments. There are three major areas that have been considered when using antennas to compensate for the impairments of the mobile radio channel:

- Noise suppression through antenna gain and effectivity

- Temporal fading and interference suppression through multiport antenna diversity

- Spatially selective interference suppression through adaptive antenna diversity

It has been known since 1927 that diversity schemes help to enhance the performance of a wireless system. There are several diversity techniques:

- Space diversity

- Polarization diversity

- Angle diversity

- Frequency diversity

- Time diversity In addition, there are several combining techniques $[11,12]$ :

- Selective

- Switched

- Maximal ratio

- Equal gain

Millimeter Waves - During recent years, millimeter waves have gained increasing interest because of bandwidth scarcity; therefore, study of millimeter-wave communication systems has drawn the attention of many researchers [14, 15]. Within the European Cooperation in the Field of Scientific and Technical Research (COST) program, the promising features of millimeter waves ( $\mathrm{mm}$ waves) for communication applications are investigated in the COST 231 project, "Evolution of Land Mobile Radio" (including personal communications). The low mm-wave band from $20-60 \mathrm{GHz}$, which is nearly unused and allows for large bandwidth applications, combines the advantages of IR (enough free bandwidth) and ultra high frequency (UHF) (good coverage). Systems operating particularly in the $60 \mathrm{GHz}$ frequency band can have a small reuse distance because of oxygen absorption at the rate of $15 \mathrm{~dB} / \mathrm{km}$. However, the indoor radio channel shows adverse frequency-selective multipath characteristics due to the highly reflective indoor environment, which results in severe signal dispersion and limits the maximum usable symbol rate. Another advantage is the fact that this frequency region is not in use by any other communications medium, so every channel can be allocated a large bandwidth: $100 \mathrm{MHz}$ channels can be used without any bandwidth problems. A third advantage of mm-wave technology is that antenna sizes are very small, so the equipment will be convenient. The fourth advantage is that the mm-wave spectrum has the potential to support broadband service access, which is especially relevant because of the advent of the broadband integrated services digital network (B-ISDN).

A major drawback of this frequency region is the fact that the technology for transmitters and receivers is not yet fully developed. As a consequence the hardware will be expensive in the early stages. It is worth mentioning that no definitive evidence has been shown to date of any hazards to the general public arising from prolonged exposure in fields of less than $10 \mathrm{~mW} / \mathrm{cm}^{2}$ in the $\mathrm{mm}$ waves.

Within the European research program, the mm-wave spectrum has been selected for the development of MBS [16]. MBS typically addresses services above $2 \mathrm{Mb} / \mathrm{s}$. The high date rates envisaged for MBS require operation at much higher frequencies, currently taken to be in the $60 \mathrm{GHz}$ bands. Study of MBS needs a lot of investigation, such as propagation modeling, antenna diversity, technology development, and so on.

Interference - Wireless systems face several types of interference (co-channel, adjacent-channel, intersymbol, simulcast, etc.) $[11,12]$. A detailed study of these interference types is necessary.

Microwave Networks and Integrated Circuits (MNs and MICs) - MNs and MICs play a role of fundamental importance in the design and development of wireless communication systems.

Design Parameters - Antenna locations, antenna spacing, antenna heights, antenna configuration, noise environment, field strength, and frequency dependency are the factors to investigate for the base station and receiver unit [11].

\section{CONCLUSIONS}

$T$ he research and investigations in the field of microwave technology will make a significant contribution in achieving the objectives of the global village. The microwave community has to meet the following research challenges related to the future wireless personal communication systems:

- Propagation modeling

- Antenna and diversity

- Millimeter waves

- Interference

- Microwave integrated circuits

- Design parameters

With the combined effort of the microwave, wireless, and networking community it is expected that the target for completing the objectives of FWPC by 2000 will be met. FWPC will provide communication services for "any person" "any- 
where" in a "multimedia environment" by "one pocket-sized unit" using a "personal reference number."

\section{REFERENCES}

[1] R. Prasad and B. Lal, "The Design of Coupled Microstrip Directional Coupler Using Teflon Substrate," JIETE, vol. 23, Aug. 1977, pp. 501-3.

[2] R. Prasad, D. Kalluri, and S. B. Sataindra, "Rectangular Waveguide Filled with Warm Isotropic Drifting Plasma," IEEE Trans. Plasma Sci., vol. 13 Sept./Oct. 1985, pp. 340-45.

[3] D. Kalluri, R. Prasad and S.B. Sataindra, "Experimental simulation of plasma using strip medium," IEEE Trans. Microwave Theory Technique, vol. 34, July 1986 , pp. $825-828$

[4] R. Prasad and J.C. Arnbak, "Enhanced Throughput in Packet Radio Channel with Shadowing," Elect. Letters, vol. 24, Aug. 1988, pp. 986-88.

[5] R. Prasad and J. C. Arnbak, "Enhanced Packet Throughput with Radio Channels with Fading and Shadowing," Proc. Canadian Conf. Electrical \& Comp. Eng., Vancouver, Canada, Nov. 1988, pp. 78-80.

[6] O. E. Dunlop, Marconi the Man and his Wireless, New York: Macmillan, 1937.

[7] W. P. Jolly, Marconi, London: Constable, 1972.

[8] R. Prasad, Code Division Multiple Access for Wireless Personal Commu nication Networks, Boston-London: Artech House, 1996.

[9] J. A. Schwarz da Silva and B. E. Fernandes, "The European Research Program for Advanced Mobile Radio Systems," IEEE Pers. Commun., vol. 2, Feb. 1995, pp. 14-19.

[10] P. W. Baier, P. Jung. and A. Klein, "Taking the Challenge of Multiple Access for Third Generation Cellular Mobile Radio Systems - A European view," IEEE Commun. Mag., vol. 34, Feb. 1996, pp. 82-89.

[11] W. C. Y. Lee, Mobile Communication Design Fundamentals, New York John Wiley \& Sons, 1993.
[12] W. C. Jakes, Jr., Microwave Mobile Communications, New York: John Wiley \& Sons, 1974

[13] J. Bach Andersen, T. S. Rappaport, and S. Joshida, "Propagation Measurements and Models for Wireless Communications Channels," IEEE Commun. Mag., vol. 33, Jan. 1995, pp. 42-49.

[14] R. Prasad and L. Vandendorpe, "An Overview of Millimeter Wave Indoor Wireless Communication System," Proc. 2nd Int'l. Conf. Universal Pers. Commun., Ottawa, Canada, 1993, pp. 885-89.

[15] E. Damosso and G. de Brito. "COST 231 Achievements as a Support to the Development of UMTS: A Look into the Future," IEEE Commun. Mag., vol. 34, Feb. 1996, pp. 90-96.

[16] L. M. Correia and R. Prasad, "An Overview of Wireless Broadband Communications," IEEE Commun. Mag., vol "35, Jan. 1997, pp. 28-33.

\section{BIOGRAPHY}

RAMJEE PRASAD has been with the Telecommunications and Traffic control Systems Group, Delft University of Technology (DUT). The Netherlands, since February 1988. He is head of the Wireless Transmission Research Section of the IRCTR (International Research Center for Telecommunications - Transmission and Radar), where he is involved in the area of personal indoor and mobile radio communications (PIMRC). He is currently involved in the European ACTS project FRAMES (Future Radio Wideband Multiple Access Systems) as a project leader of DUT. He has published over 200 technical papers, and a book entitled CDMA for Wireless Personal Communications (Artech House). His current research interests are in the areas of packet communications, multiple access protocols, adaptive equalizers, spreadspectrum CDMA systems, and multimedia communications. He is the Coordinating Editor and one of the Editors-in-Chief of a Kluwer international journal, Wireless Personal Communications, and also a member of the editorial boards of other international journals, including IEEE Communications Magazine. 